\title{
The trajectories of depressive symptoms expressed in early childhood differ between boys and girls
}

By Dr Jessica K Edwards

A study by Diana Whalen and colleagues at Washington University has used latent class analysis (LCA) to identify and define the trajectories of latent classes of depressive symptoms in early childhood. The study included 348 children from the Preschool Depression Study, who across a 10-year period completed at least three behavioural assessments. The researchers assessed symptoms of depression and oppositional defiant disorder/conduct disorder (ODD/CD) diagnoses using the Preschool Age Psychiatric Assessment (PAPA), as well as social risk factors, family history of affective disorders and functional impairment. The researchers identified three trajectories of depressive symptoms based on high, medium and low symptom severity in boys and girls. Interestingly, the high depression severity latent class in boys was characterized by an increase in symptom severity from preschool through to school age, and then a decline during later school age. For girls, however, the high depression severity latent class was characterized by stable symptom severity over time. Early childhood social adversity, family history of affective disorder, preschool onset of ODD/CD and school age functional impairment differentiated the high-risk trajectory classes among boys and girls. Finally, the researchers reported that early childhood social adversity prior to age 5 could predict greater depressive symptom severity across preschool and into school age in girls and boys.
Whalen, D.J., Luby, J.L., Tilman, R., Mike, A., Barch, D. \& Belden, A.C. (2016), Latent class profiles of depressive symptoms from early to middle childhood: predictors, outcomes, and gender effects. J Child Psychol Psychiatr. 57: 794-804. doi:10.1111/jcpp.12518 Glossary:

Preschool Age Psychiatric Assessment (PAPA): A parent report-only assessment for children aged 2 to 5 years. PAPA is derived from the Child and Adolescent Psychiatric Assessment (aimed at children aged 9 to 18 years) but has been substantially revised in interview content and structure to be relevant to children of a very young age. PAPA includes all DSMIV criteria relevant to young children.

Latent class analysis (LCA): A modelling technique to identify unobservable, or latent, subgroups within a population 